Editorial

Z Herz- Thorax- Gefäßchir 2020 · 34:329-330 https://doi.org/10.1007/s00398-020-00402-1

(c) Springer Medizin Verlag GmbH, ein Teil von Springer Nature 2020

Liebe Leserinnen und liebe Leser,

einige von Ihnen mögen sich noch an die groteske Situation erinnern:

Im Rahmen einer internationalen Jahrestagung einer medizinischen Fachgesellschaft wird aus einem Hybrid-OP eine TAVI live in das größte Auditorium der Veranstaltung übertragen. Der Operateur beendet den Eingriff, stellt sich vor den Kathetertisch, auf dem der Patient noch liegt, und beantwortet Fragen aus dem Publikum. Im Hintergrund sieht man währenddessen, unbemerkt vom Operateur, dass das Team um den Anästhesisten den soeben operierten Patienten mit einer Herzdruckmassage reanimiert.

Der Ausgang der Behandlung ist mir nicht bekannt; auch bei mir selber stellte ich einen gewissen Voyeurismus fest, indem ich von dem Drama gefesselt war und das Outcome des Patienten in den Hintergrund rutschte.

Um zu vermeiden, dass solche Szenen in öffentlichen Medien die zweifelslos vorhandene Sensationsgier in der breiten Masse an Zuschauern befriedigen können, hat sich die Deutsche Gesellschaft für Chirurgie in einem Positionspapier deutlich gegen Live-Übertragungen von Operationen vor einem Laienpublikum, insbesondere im Fernsehen, ausgesprochen. Dieses Positionspapier ist in dieser aktuellen Ausgabe der Zeitschrift für Herz-, Thorax- und Gefäßchirurgie nachzulesen. Anlass für dieses Positionspapier war kürzlich das Herantreten von Fernsehsendern an herzchirurgische Kliniken in Deutschland mit dem Wunsch,

Klaus Kallenbach

Haerzzenter - INCCI, Luxembourg, Luxemburg

\title{
Live-Übertragungen von Operationen: etabliertes Weiterbildungsinstrument oder Patientengefährdung?
}

Herzoperationen live übertragen zu dürfen. Lockmittel ist dabei sicherlich, dass der Bekanntheitsgrad der Klinik erhöht wird; aber man male sich aus, was passieren würde, wenn eine lebensbedrohliche Komplikation live gesendet würde - vor Laien und der aufmerksamen Regenbogenpresse ...! Die eindeutige Konsequenz, auch im Positionspapier: keine Live-Übertragungen von Operationen vor einem Laienpublikum!

Die Frage, die bleibt, lautet: Sollten Live-Operationen im Rahmen von Fachtagungen für ein ärztliches Publikum gezeigt werden? Das Positionspapier lehnt diese Praxis nicht ab, obwohl nicht ausgeschlossen werden kann, dass unter bestimmten Bedingungen der Patient durch die Live-Übertragung zusätzlich gefährdet wird. In den Annals of Thoracic Surgery erschien 2008 ein bemerkenswertes gemeinsames Positionspapier der amerikanischen herzchirurgischen Fachgesellschaften AATS und STS, welches genau diese Frage diskutierte [1]. Es wird berichtet, dass die Ethics Committees beider Fachgesellschaften empfehlen, im Rahmen der jeweiligen Jahrestagungen auf die Übertragung von LiveOperationen zu verzichten. In den Fachgesellschaften wird daraufhin diskutiert, wie die Verpflichtung zur Weiterbildung gegen eine mögliche Patientengefährdung im Rahmen von Live-Operationen gewichtet werden sollten. Dabei wird unterschieden zwischen 1.) Beobachtung von Live-Operationen (in Deutschland oft nicht möglich, in den USA durch OPKuppeln üblich, s. auch Grey's Anatomy); 2.) Übertragungen von Live-Operatio- nen; 3.) Übertragung von aufgezeichneten Operationen (auch als „life in a box" bezeichnet). Alle Methoden werden ausschließlich für ein medizinisches Fachpersonal erörtert. Nach ausführlicher Diskussion von Für und Wider kommt das gemeinsame Komitee aus STS und AATS unter anderen zu folgenden Empfehlungen: a) Beobachtung von Live-Operationen $\mathrm{zu}$ Weiterbildungszwecken ist eine akzeptierte Methode; b) aufgezeichnete Übertragungen sind Live-Übertragungen $\mathrm{zu}$ bevorzugen; c) nationale und internationale kardiochirurgische Fachgesellschaften sollten erwägen („,consider“), Live-Übertragungen auf ihren Jahrestagungen vor einem großen Publikum zu verbieten; d) Chirurgen sollten sich grundsätzlich nicht an Live-Übertragungen von Operationen in der Öffentlichkeit beteiligen.

Sie merken, das Thema ist komplex, und ich rate Ihnen zur Lektüre des Positionspapieres. Meinungsbildung ist auch Teil des Auftrages unserer Zeitschrift, zumindest aber die Anregung von kontroversen Gedanken und Diskussionen. Meine persönliche Meinung, die ich mir als Verfasser des Editorials erlaube kundzutun, ist eindeutig: „Live in a box“, am besten nachbearbeitet unter didaktischen Gesichtspunkten, ist der LiveÜbertragung von Operationen vorzuziehen. Aus eigener Erfahrung weiß ich, wie sich das übertragene Live-Operieren vor einem kritischen Publikum anfühlt, und kann alle in dem amerikanischen Positionspapier angeführten Argumente gegen die Übertragung von LiveOperationen nachvollziehen. 
Ihnen, lieben Leserinnen und Lesern, wünsche ich viel Spaß bei der Lektüre der aktuellen Ausgabe der Zeitschrift für Herz-, Thorax- und Gefäßchirurgie!

Herzlichst,

Ihr

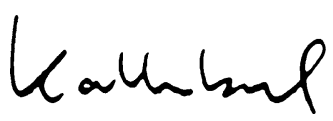

Klaus Kallenbach

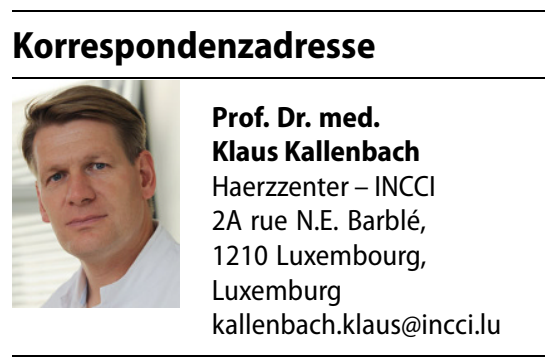

Interessenkonflikt. K. Kallenbach gibt an, dass kein Interessenkonflikt besteht.

\section{Literatur}

1. Sade RM, American Association for Thoracic Surgery Ethics Committee, Society of Thoracic Surgeons Standards and Ethics Committee (2008) Broadcast of surgical procedures as a teaching instrument in cardiothoracic surgery. Ann Thorac Surg 86(2):357-361. https://doi.org/10.1016/j. athoracsur.2008.05.066

\section{Hübler, Matthias (Hrsg.) Komplikationen in der Inten- sivmedizin}

\section{Fallbeispiele - Analyse - Präven- tion}

Heidelberg: Springer-Verlag 2019, 412 S., 93 Abb., (ISBN: 978-3-66258307-4), Softcover 44,99 EUR

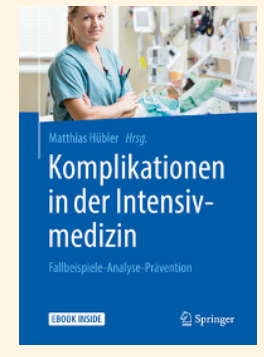

Nach ihrem Erfolg mit "Komplikationen in der Anästhesie" haben Matthias Hübler und sein Autor:innen-Team aus Dresden nun einen Nachfolger mit dem Titel „Komplikationen in der Intensivmedizin" herausgebracht. Die Rezeptur ist unverändert: $\ln 24$ Fällen wird die Welt der Intensivmedizin aufgeblättert, alle 24 direkt aus dem wirklichen Leben gegriffen. Es gibt Ärzte und Ärztinnen wie Du und ich mit ihren Stärken und Schwächen, Pfleger und Pflegerinnen wie wir sie schätzen - überwiegend mehr, selten weniger -, und es gibt Patienten und Patientinnen mit ganz normalen, häufigen Erkrankungen

- Intensivmedizin eben. Auf einmal aber passiert etwas Unerwartetes, dann noch etwas manchmal eine ganze verhängnisvolle Kette. Und immer war es nicht einfach nur Schicksal, sondern die handelnden Personen waren Teil des Unheils: Sie haben etwas übersehen, nicht richtig gedeutet, falsch reagiert, sind einer technischen Tücke erlegen oder in eine organisatorische Falle getappt. Auch hier wird nichts konstruiert, sondern alles ist wiederum aus dem wirklichen Leben gegriffen. Matthias Hübler schöpft als langjähriger Intensivmediziner und Protagonist des Critical Incident Reporting System (CIRS) aus einem schier unerschöpflichen Vorrat von kleineren und größeren Fehlern, die sich alle genau in dieser Weise wirklich schon einmal ereignet haben - und zwar nicht etwa selten, sondern als wiederkehrender Teil des intensivmedizinischen Alltags. Und er kennt die Ursachen, die so vielfältig sind wie die Fehler selbst. Oft genug sind psychologische Mechanismen eine treibende Kraft. Mit jedem Fall, den man liest, wird klarer: Dies sind nicht die Fehler der anderen, sondern die Fehler von Dir und mir, von uns allen.
Umso bemerkenswerter ist die konsequente Haltung, mit der das Buch dem Alltagsfaktor Fehler begegnet. Nicht der erhobene Zeigefinger führt durchs Thema, an keiner Stelle wird moralisiert. Ausgehend von der schlichten Feststellung, dass Fehler vorkommen, werden sie benannt, wird ihr Mechanismus analysiert, und werden Hinweise gegeben, wie sie in Zukunft vermieden werden können Die Fälle folgen immer demselben Aufbau. Im ersten Teil wird die Geschichte erzählt und mit fachlichen Informationen zu Krankheiten, Medikamenten und Prozeduren angereichert. Im zweiten Teil der Darstellung geht es dann eher systematisch um die Fehler, die Teil der Geschichte waren. Der Stil ist locker und unterhaltsam, ein kleiner Hauch des guten alten „House of God" weht herein, Manches dabei ist vielleicht etwas verspielt. Die Sachlichkeit der Darstellung und der Ernst des Anliegens gehen aber nie verloren. Nach weniger als einer halben Stunde ist man durch mit einem Fall. Man wurde ertappt, verständnisvoll auf den Fehler und seinen Mechanismus aufmerksam gemacht und ist motiviert es in Zukunft besser zu machen. Obendrein hat man fachlich einen kleinen Happen dazugelernt. Auf diese Weise ist das Buch ein guter Reisebegleiter oder das ideale Nachtschränkchen-Utensil, sei es zu Hause oder im Dienst. Am besten aber liegt es auf der Intensivstation an einem für alle zugänglichen Platz - es sage keine:r, dass sich nicht dann und wann doch einmal zwanzig Minuten für eine kleine geistige Erfrischung finden.

Dem Buch ist hoffentlich der verdiente Erfolg beschieden, so dass man bei einer zweiten Auflage die kleinen Fehler beseitigen kann, die sich immer wieder in die Grammatik, und ganz manchmal in - vorwiegend internistische - Inhalte eingeschlichen haben.

Prof. Dr. med. Sebastian M. Schellong, Dresden 